HC (15 studies, 538 patients, 416 HC, MD: -2.24 (95\% CI -4.12, $-0.36), p=0.02, I 2=64 \%)$. There were no statistically significant differences between patients and controls in the other three outcomes. CBFv (cm/s): 6 studies, 305 patients, 198 HC, MD: -1.23 (95\% CI $-6.10,3.64, \mathrm{p}=0.62, \mathrm{I} 2=65 \%$. Combined $\mathrm{CBF}$ and CBFv: 20 studies, 804 patients, 573 HC, SMD: -0.16 (95\% $\mathrm{CI}-0.32,0.01), \mathrm{p}=0.06 \mathrm{I} 2=51 \%$. Ratio of uptake of radiotracer: 3 studies, 60 patients, $53 \mathrm{HC}$, MD: -0.11 (95\% CI $-0.11,0.11$ ), $\mathrm{p}=1.00, \quad \mathrm{I} 2=0 \%)$. The narrative synthesis revealed varying results, with many studies identifying a decrease in CBF in depressed patients compared to controls, but other studies identifying an increase, or mixed results. Multiple regions of impairment were identified, including the anterior cingulate cortex and prefrontal cortex.

Conclusion. There was a statistically significant reduction in CBF in depressed patients compared to controls. The narrative synthesis revealed varying results, however specific regions of interest have been identified. Further research is needed to explore the effect of antidepressant medication, utilising different imaging modalities, and at different levels of disease severity.

\section{Coping with a modern pandemic-an online survey of Anesthesiologists in India during COVID-19}

Punitha Chockalingam $^{1 *}$, Kalpana Balakrishnan ${ }^{1}$, Priyadarshini Natarajan ${ }^{2}$, Surendran Veeraiah ${ }^{1}$, Revathy Rajagopal ${ }^{3}$ and Vinodh Kumar Elumalai ${ }^{1}$

${ }^{1}$ Cancer Institute (WIA), Adyar; ${ }^{2}$ South London and Maudsley NHS Foundation Trust and ${ }^{3}$ HCG Cancer Hospital

${ }^{\star}$ Corresponding author.

doi: 10.1192/bjo.2021.652

Aims. In 2020, India was one of the worst affected countries by COVID-19. As the pandemic spread, creating undue pressure on health care workers (HCWs), there was an urgent need for the development of appropriate interventions to protect their mental health. This study aims to study the effect of COVID-19 on the mental health of anaesthesiologists in India and factors that influence their coping behaviour.

Method. The study was designed as a semi-structured, descriptive, cross-sectional, online open survey and conducted on Google forms between 21st May and 20th June 2020, among practicing anaesthesiologists across India. The participants were recruited by sending messages to their emails and through social media platforms. It created a small number of international respondents, who were also included (India $=301$, rest $=23$ ). The self-designed questionnaire had 30 questions in the form of multiple choices, checkboxes, linear scales and short comments. Informed consent was recorded at the outset. Details such as demographic characteristics, place and nature of work, pandemic related changes in duration or pattern of work, psychological symptoms during and after working hours, fears about quarantine, were collected in the survey. Statistical Analysis was performed using Statistical Package for Social Sciences (SPSS Statistics for Mac Version 21.0 IBM Corp., USA)

Result. Among the 324 participating anaesthesiologists, a prevalence rate of $64.8 \%$ for stress, $51.2 \%$ for anxiety and $65.7 \%$ for depression was noted, which was double the rate from prepandemic studies. Those between the ages of 30 and $50(p=$ 0.010 OR:2.191) and working in government run $(\mathrm{p}=0.045$ OR:2.564) COVID-19 hospitals in India ( $p=0.002$ OR:2.018), were particularly stressed $(33.3 \%)$ and anxious $(38 \%)$ than the rest. Increased workload, contracting the virus and becoming an infectious source to their family (88.6\%) were their prime concern. Formulating standard operating procedures (SOP) (66.7\%) and procuring personal protective equipment (PPE) (56.2\%) were some of the challenges faced at work. Most of them recommended a congenial workplace $(68.8 \%)$ and family support $(60.8 \%)$ to help them work through their anxiety and fear, while a few reported considering leaving their career $(34.8 \%)$ from fear of monetary loss and burn out (53.8\%).

Conclusion. COVID-19 has changed the professional and personal life of anaesthesiologists in India. Irrespective of their workplace, their fears and challenges remain universal. Early identification of anxiety and depression and providing appropriate psychological support will prevent deep and enduring damages to the lives of these professionals.

\section{Taking leadership over psychopathogenic} environments

\author{
Alastair Cockburn ${ }^{1 \star}$ and Jane Morris ${ }^{2}$ \\ ${ }^{1}$ Royal Infirmary of Edinburgh and ${ }^{2}$ Royal Cornhill Hospital \\ ${ }^{*}$ Corresponding author.
}

doi: 10.1192/bjo.2021.653

Aims. Do psychiatrists believe children are growing up in psychopathogenic environments that significantly contribute to mental illhealth? If so, do they feel empowered to change those environments? If not, how can psychiatrists be given a role where they can create meaningful change? Finally, how much responsibility can psychiatry usefully take for changing psychopathogenic environments?

Background. We define psychopathogenic environments as environments that predispose to mental ill-health. It is the psychological environment we live in - including income, the way we interact with others (e.g. social media, bullying), what we do with our free time, pressures at school and expectations of our peers. It is not discrete events (e.g. trauma) and stretches beyond life at home (where many ACE's occur).

Self-harm presentations to medical professionals amongst teenagers are on the rise, Universities report a fivefold increase in disclosure of mental health conditions in the last decade. Here we consider if psychopathogenic environments are part of the cause of these changes.

Method. A 10-item questionnaire distributed to Child and Adolescent Psychiatrists in NHS Lothian, NHS Grampian and Manchester University NHS Trust via a consultant in each Trust. Result. All 14 respondents said psychopathogenic environments are "very important" contributors to mental ill-health. 13/14 say the environments have got worse in the last 10 years. 13/14 responded negatively about whether psychiatrists could change them. When given white space to tackle the problem they suggested changes were needed from Government including against poverty / inequality, education, public health nudges, more resources, MDT working and better access to leisure facilities. Given specific choices, 11/14 identified influencing Government as a major way forward. Conclusion. This group of psychiatrists believe psychopathogenic environments are; 1) a very important contributor to mental ill-health 2) getting worse but 3) feel largely powerless to tackle it. It is a problem they think is important and want to engage in, but lack time, resources and struggle with the complexity of the problem. How therefore, can psychiatrist show leadership in this area? The two perspectives to consider how to empower psychiatrists to help create change are 1) how they can influence the environment for individual patients, 2) how they can influence public policy and government to make wider changes.

Is this the job of psychiatrists? Not alone, but as agents they have a unique insight and authority as both a lens for and director of these environments. 